\title{
INFLUENCE OF THE COMPOSITION EVOLUTION OF WASTE COMPUTER MOTHERBOARDS ON THEIR RECYCLING STRATEGY
}

\author{
SORIN-AUREL DORNEANU ${ }^{a, b}$, ALEXANDRU-ANDREI AVRAM ${ }^{a}$, \\ ALEXANDRU-HORAȚIU MĂRINCAŞ ${ }^{a}$, NICOLETA COTOLAN ${ }^{a}$, \\ TIBERIU FRENŢIU' ${ }^{a}$, PETRU ILEA ${ }^{a}$, , *
}

\begin{abstract}
Even if the mobile devices gain more popularity comparing to the desktop personal computers (DPCs), the last ones are still intensively used where highly computing or storage capabilities are required. Because the replacing rate of DPCs are very high, the amount of obsolete equipments is also huge, reclaiming adequate efficient and ecological recycling techniques. In this field, the main efforts are focused on the DPCs motherboards (DPCMBs) recycling because, even if they represent only 5 to $10 \%$ from the DPCs weight, they concentrate up to $80 \%$ from the recoverable value. By accounting the DPCMBs' traditional and incipient recycling technologies, it is obvious that none of them can solve alone the problem of this waste type. In this context, during the present work, the composition evolution of 10 DPCMBs released between 1998 and 2008 was evaluated and analyzed, the obtained results being used to design a feasible and eco-friendly combined strategy for integral DPCMBs recycling.
\end{abstract}

Keywords: Waste printed circuit boards, metals recovery, composition evolution, recycling strategy, eco-friendly process

\section{INTRODUCTION}

Obviously, the development of the modern society cannot be imagined without the support of the advanced computing systems. Among them, the portable devices (i.e. laptops and tables) achieve an increased popularity comparing to the desktop PCs (DPCs), with more than 162, 150 and 94 million units, respectively, forecasted to be shipped in 2018 [1]. Even if the costs of

a Babeş-Bolyai University, Faculty of Chemistry and Chemical Engineering, 11 Arany Janos Str., RO-400028, Cluj-Napoca, Romania

b Babeş-Bolyai University, Interdisciplinary Research Institute on Bio-Nano-Sciences, 42 Treboniu Laurian Str., RO-400271, Cluj-Napoca, Romania

*Corresponding author: pilea@chem.ubbcluj.ro 
laptops manufacturing and exploiting are sensibly lower over those of DPCs [2], the last ones are still extensively used in fields where highly computing or storage capabilities are required (e.g. servers, data bases, graphical stations, processes control, research, etc.) and their development and replacing rate are very high [3]. Therefore, the amount of the resulting DPCs waste remains significant, requiring adequate efficient and ecological recycling techniques [4]. From the wasted DPCs systems, the central processing units (CPUs) represent the major component and include the main board, also known as motherboard. The DPCs motherboards (DPCMBs), representing between 5 and $10 \%$ of the DPCs weight [5] are considered the most economically interesting components due to the high value of the recoverable metals [6-8]. Despite the fact that the total amount of the precious metals (especially $\mathrm{Au}$ ) used for DPCMBs manufacturing represents less than $1 \%$ from the boards' weight, these metals concentrate more than $80 \%$ of the recovered value [5]. As a consequence, intensive researches were dedicated to the DPCMBs recycling, being proposed various processes, e.g. physico-mechanical [7, 9], hydrometallurgical [6, 10, 11], pyrometallurgical [12,13], pyrolytic [14] biometallurgical [15] or combination of thereof as presented in research papers [9, 16] or exhaustive reviews [17-19]. In spite of the intense efforts, confirmed by the high number of published papers [20] and patents [21], the great majority of the proposed technologies presents serious disadvantages, e.g. generation of large inter-contaminated metallic / nonmetallic fractions during the physico-mechanical treatment [22], high raw materials consumption and generation of large wastewater volumes for the hydrometallurgical approach, high energy consumption, low selectivity and generation of toxic gases during the high temperature treatments [12, 14, 23]. The true electrochemical recycling of metals from DPCMBs, that must include compulsory (i) the metals electrodeposition and (ii) the simultaneously electrochemical recycling of the leaching agent [24-27] and/or metals anodic dissolution [28], starts to represent a feasible alternative from economical [29] and ecological [30] points of view, allowing also a significant minimization of the disadvantages pointed before. Moreover, the efficiency of the DPCMBs' electrochemical recycling can be improved by the process intensification [31] or use of special electrolytes, like ionic liquids [32].

Unfortunately, due to the high heterogeneity, complexity and nonuniformity of the DPCMBs, the traditional or new-born recycling technologies mentioned before may not meet the future industry requirements because of environmental contamination, high cost, low efficiency and/or generation of new waste fluxes. Furthermore, a single technology still has several limitations and cannot solve all the DPCMBs' recycling problems, requiring combinations of more than one process or technology [33]. Practically, because the great majority of the cited technologies are focused mainly on metals recovery and high profitability, the problem of the nonmetallic parts (NMPs) recycling is 
neglected or treated inadequately. For example, in the pyrometallurgical processes, the NMPs are considered as supplementary fuel and, consequently, their recycling rate is, practically, $0 \%$ [34]. Similarly, the physico-mechanical approach leads to highly inter-contaminated metallic and nonmetallic fractions, the last ones containing variable amounts of plastics, epoxy resins, glass, fiberglass, ceramics and, most often, extremely toxic compounds like heavy metals traces and flame retardants [35]. Therefore, these new generated waste fluxes, mainly as powders, are very difficult to recycle or dispose.

In this context, in the present work, we decided to study the evolution of the DPCMBs composition aiming to propose an optimal scenario for their integral recycling. Our demarche was justified by the fact that, to the best of our knowledge, the temporal evolution of the metals content in whole DPCMBs was not yet studied, a similar work being focused only on the $\mathrm{Cu}$ and $\mathrm{Au}$ content of RAM modules [36]. In our case, 10 obsolete DPCMBs released between 1998 and 2008 were selected and manually pre-treated in order to remove several unwanted components: the $\mathrm{Li}$ batteries (LiBs), chipset aluminium heat sinks (AHS), brass screws (BS) derived from the D-sub connectors and the cylindrical aluminium electrolytic capacitors (EC). Afterwards, the exposed metals from the DPCMBs were dissolved using the aqueous $\mathrm{Br}_{2} / \mathrm{KBr}$ leaching system and the resulting solutions were analyzed by ICP-OES. Finally, based on the weight balance of the all contained materials and the literature data, an original flowchart for the complete DPCMBs recycling was elaborated.

\section{RESULTS AND DISCUSSION}

Because the profitability of a recycling technology represents a key factor for a successfully subsequent industrial implementation, the amount and the nature of all the recovered materials during the present work were rigorously evaluated and accounted. In our opinion, as pointed in a previous work [26], in order to reduce the recycling cost and to avoid undesirable phenomenon like hydrogen evolution, explosion risks and electrolyte contamination, it is preferable to remove some components (LiBs, AHS, BS and EC) from the DPCMBs before the main leaching step. Consequently, in the first step of our study, these unwanted parts were manually removed from each board and weighed separately, the obtained values being presented in Figure 1 as $\mathrm{kg}$ per ton of DPCMBs waste. Because the weight of the all removed LiBs was the same, of around $3 \mathrm{~g}$ each, it was not included in this comparison. Concerning the other parts, their amounts present a random distribution, without any visible increasing or decreasing tendency. Consequently, for further estimations, the mean amount values were considered. 


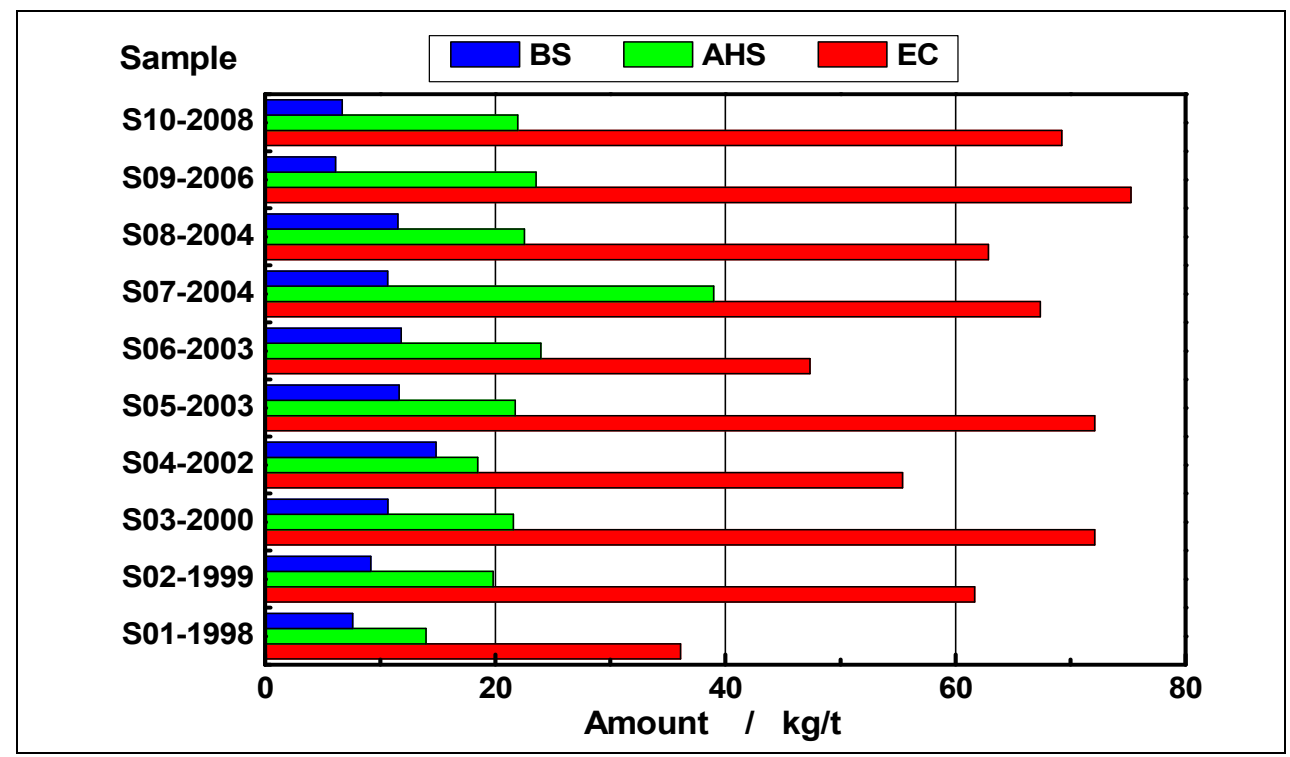

Figure 1. Amount evolution of the manually removed components from 10 studied DPCMBs

Based on data presented in Figure 1, mean amounts of 10.1, 22.7 and $61.9 \mathrm{~kg} / \mathrm{t}$ of DPCMBs were calculated for BS, AHS and EC, respectively. For an electric energy price of $0.133 € / \mathrm{kWh}$ [37] and a recycling energy consume of around $6.6 \mathrm{kWh} / \mathrm{kg}$ of BS (for brass leaching and metals electroextraction), an economy of $\sim 9 € / \mathrm{t}$ of DPCMBs can be obtained only by direct BS valorization. Similarly, considering energy consumptions of $12 \mathrm{kWh} / \mathrm{kg}$ for $\mathrm{Al}$ leaching and $12.5 \mathrm{kWh} / \mathrm{kg}$ for Al electroextraction, the direct valorization of aluminium scrap included in the AHS and EC (at least $80 \mathrm{~kg} / \mathrm{t}$ of DPCMBs) can generate an economy of $260 € / t$ of DPCMBs.

After the pre-treatment step described before, the metals from DPCMBs were dissolved using the aqueous $\mathrm{Br}_{2} / \mathrm{KBr}$ leaching system, liberating all the components from the boards surface. Subsequently, the leaching solutions were decanted, and the resulting solid parts were washed, dried and manually sorted on the following categories: (i) waste printed circuit boards (WPCBs), (ii) plastic parts (PPs), (iii) electronic chips and surface mounted devices (C\&SMDs) and (iv) magnetic parts and undissolved metals (M\&UMs). Each set of solid parts were weighed individually and the total amounts of the dissolved metals were evaluated by calculating the difference between DPCMBs mass before and after the leaching step. The resulting values are presented in Figure 2. 


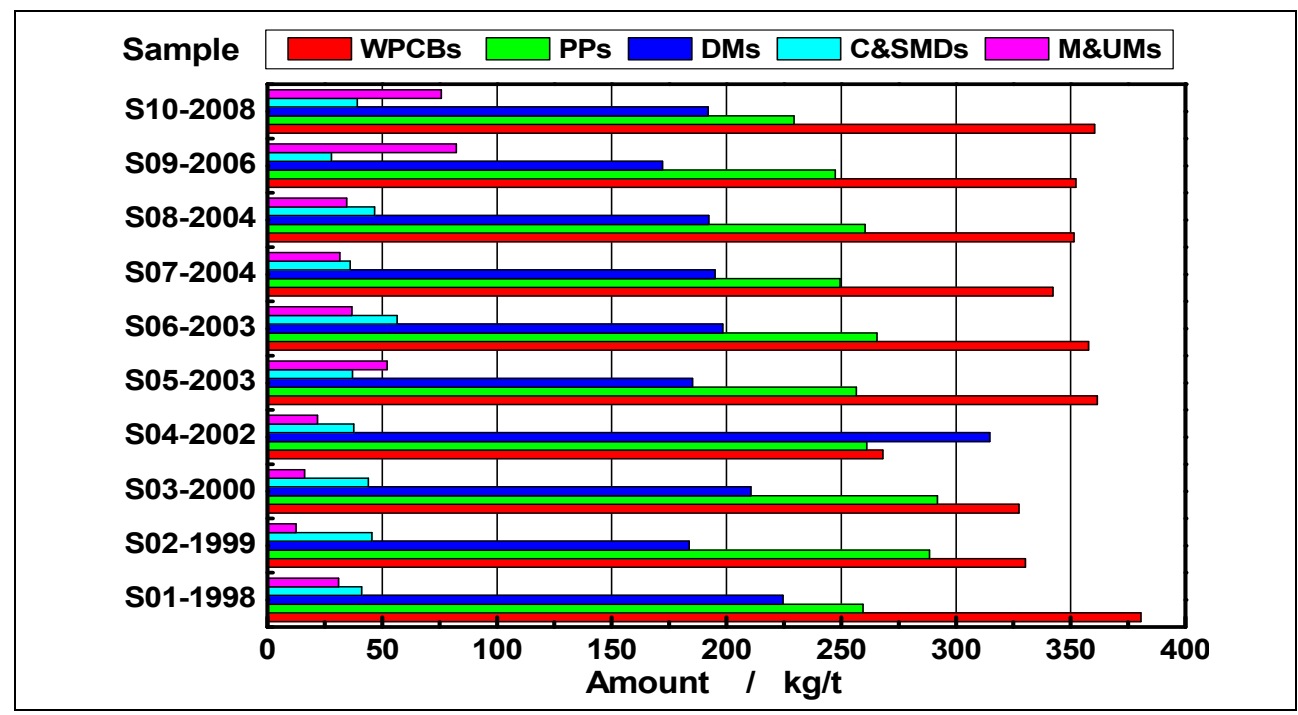

Figure 2. Amount evolution of the solid parts and total dissolved metals from 10 studied DPCMBs after the leaching step

As it can be seen from Figure 2, the amounts of solid parts and DMs presents also a random distribution, without any coherent evolution. As a consequence, for the further estimations, the mean amount values were considered.

The comparison between the mean amounts of the separated fractions, presented in Figure 3, reveal that the total quantity of WPCBs and PPs represents more than $60 \%$ from the initial DPCMBs weight, requiring the development of dedicated recycling strategies.

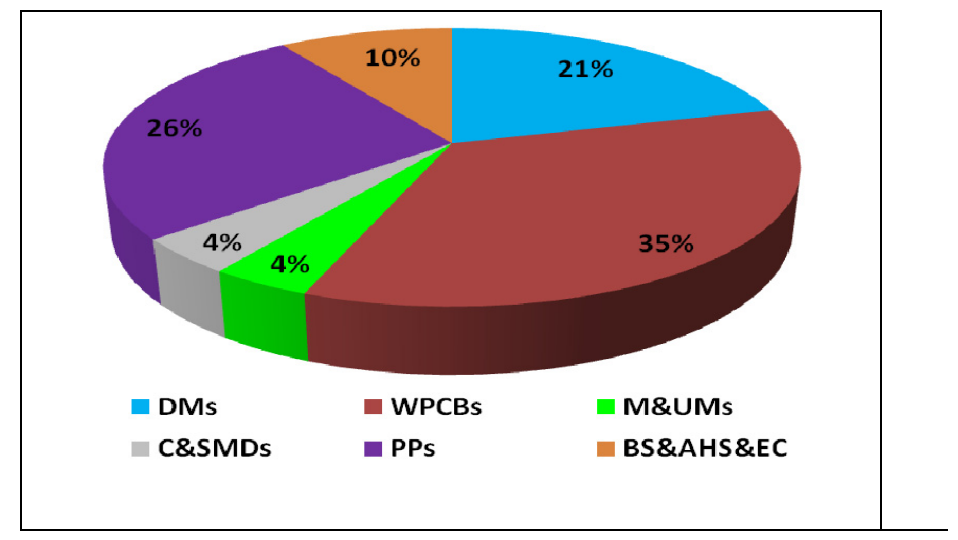

Figure 3. Distribution of the solid parts and total dissolved metals weight from 10 studied DPCMBs after the leaching step 
The evolution of the DPCMBs metals' content was evaluated by ICPOES, the obtained results being presented in Figure 4. Concerning the major (more abundant) metals amounts (see Figure 4.A), it can be observed that copper quantity presents a decrease tendency from 1998 to 2008, and, after the implementation of the UE RoHS directive in 2006, the $\mathrm{Pb}$ content decreased to 0 , simultaneously with a small raise of the Sn content.

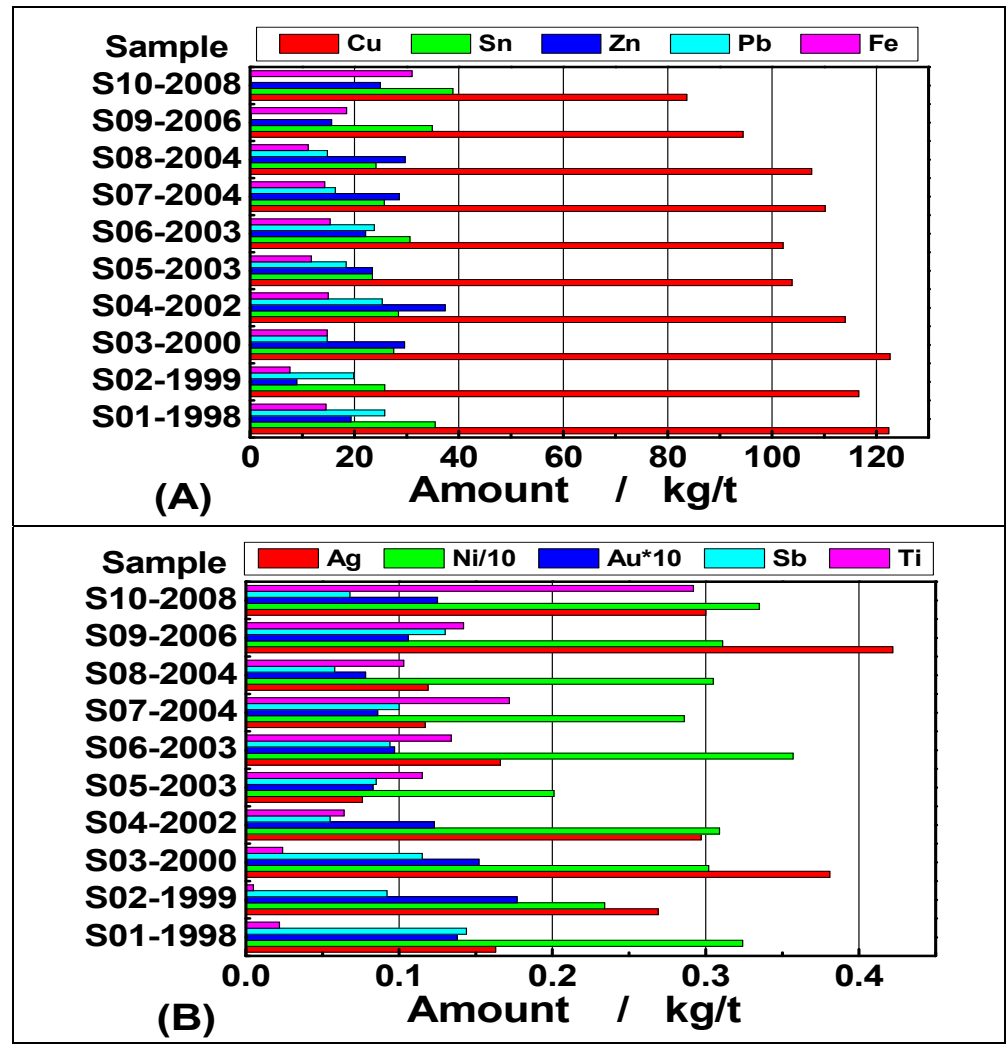

Figure 4. Amount evolution of the major $(A)$ and minor $(B)$ dissolves metals from 10 studied DPCMBs

Concerning the minor (less abundant) metals amounts (see Figure 4.B), Ti presents an general increase tendency, all the other elements remaining at relative constant levels.

Generally, excepting $\mathrm{Cu}, \mathrm{Ti}$ and $\mathrm{Pb}$, the amount of the other dissolved metals presents also a random distribution, without visible increasing or decreasing tendencies, allowing us to consider the mean values for further interpretations. 
From another point of view, it is worth to note that, until this stage, the amounts of $\mathrm{Cu}$ remaining in the WPCBs fraction and of the other base metals (BMs) and precious metals (PMs) blocked inside of the C\&SMDs parts was not evaluated. In order to solve first problem, we considered that the tested DPCMBs are of FR4 0.062' type (4 Cu layers; total thickness of $1.6 \mathrm{~mm}$ ) [38], having an average weight of $0.5 \mathrm{~kg}$ and a mean area of 500 $\mathrm{cm}^{2}$. In these conditions, we estimate that, for each ton of DPCMBs, at least $75 \mathrm{~kg}$ of undissolved $\mathrm{Cu}$ are still blocked under the epoxy layers.

Concerning the metals included in the C\&SMDs fraction, we found, after the appropriate treatment and analysis, that the amount of $\mathrm{Au}$ is $\sim 13$ times higher comparing that dissolved during first leaching step. A synthesis of the obtained mean results for one ton of DPCMBs and an estimation of the potential revenues resulting from the most valuable recovered metals are presented in Table 1 , together with a significant reference [39] concerning the metals content in the pins of DPCMBs.

Table 1. Mean amounts of the most valuable metals recovered during this work and their potential revenues

\begin{tabular}{|c|c|c|c|c|c|c|c|c|}
\hline Metals & $\mathrm{Au}$ & $\mathrm{Ag}$ & $\mathrm{Cu}$ & Sn & $\mathbf{P b}$ & $\mathrm{Ni}$ & $\mathrm{Zn}$ & Total \\
\hline Exposed mass [kg/t] & 0.012 & 0.231 & 107.7 & 29.5 & 16.0 & 2.96 & 24.0 & 180 \\
\hline Mass in WPCBs [kg/t] & - & - & 75.3 & - & - & - & - & 75.2 \\
\hline Mass in C\&SMDs [kg/t] & 0.160 & 0.011 & 7.6 & 0.39 & 0.68 & 0.41 & 0.08 & 9.33 \\
\hline Total mass $[\mathrm{kg} / \mathrm{t}]$ & 0.172 & 0.242 & 190.6 & 29.9 & 16.6 & 3.37 & 24.0 & 265 \\
\hline Price [€/kg] [40] & 34808 & 408.6 & 5.49 & 16.3 & 1.69 & 9.5 & 2.22 & - \\
\hline Total revenues [€/t] & 5970 & 99 & 1045 & 487 & 28 & 32 & 54 & 7715 \\
\hline Mass in Pins [kg/t] [39] & 0.015 & 0.037 & 55.7 & 2.54 & 0.84 & 0.82 & 16.7 & 76.7 \\
\hline
\end{tabular}

The results of an exhaustive study focused only on the metals content of the DPCMBs connectors pins (see last row of Table 1) confirmed us that the major amount of recoverable gold is entrapped inside of the C\&SMDs fraction and represents up to $80 \%$ of revenues. Moreover, in respect to the total mass of recoverable metals from DPCMBs, only $70 \%$ of $\mathrm{Zn}$ (as brass), $8.5 \%$ of Sn (as solder traces or bronze) and $29 \%$ of $\mathrm{Cu}$ (as alloys or pure metal) are included in the pins fraction, and more than $39 \%$ of $\mathrm{Cu}$ is entrapped in the WPCBs fraction. Consequently, the C\&SMDs and WPCBs fractions must be extremely carefully and efficiently treated in order to gain a maximum profit, avoiding metals loss and secondary pollution.

Finally, we can conclude that, indifferently of the DPCs age group, applying adequate technologies, a revenue of over $7000 €$ can be obtained for 1 ton of recycled DPCMBs, the metal values corresponding to the series $\mathrm{Au}>\mathrm{Cu}>\mathrm{Sn}>\mathrm{Ag}>\mathrm{Zn}>\mathrm{Ni}>\mathrm{Pb}$.

Based on applied protocols, obtained results and literature data, we succeeded to elaborate an original DPCMBs recycling flowchart able to recover all the materials included in these wastes, without generation of 
new polluting fluxes. The schematic diagram of a such technology is presented in Figure 5 and starts from the obsolete DPCs. Taking into account that the final step of DPCs manufacturing is based on manual assembling and in accordance with recent studies [34, 41], it is clear that only the manual primary dismounting can assure a complete valorization of the all included materials. Subsequently, the extracted DPCMBs must be treated manually to remove the LiBs and AHS. Concerning the EC, they can be removed manually or automatically $[42,43]$.

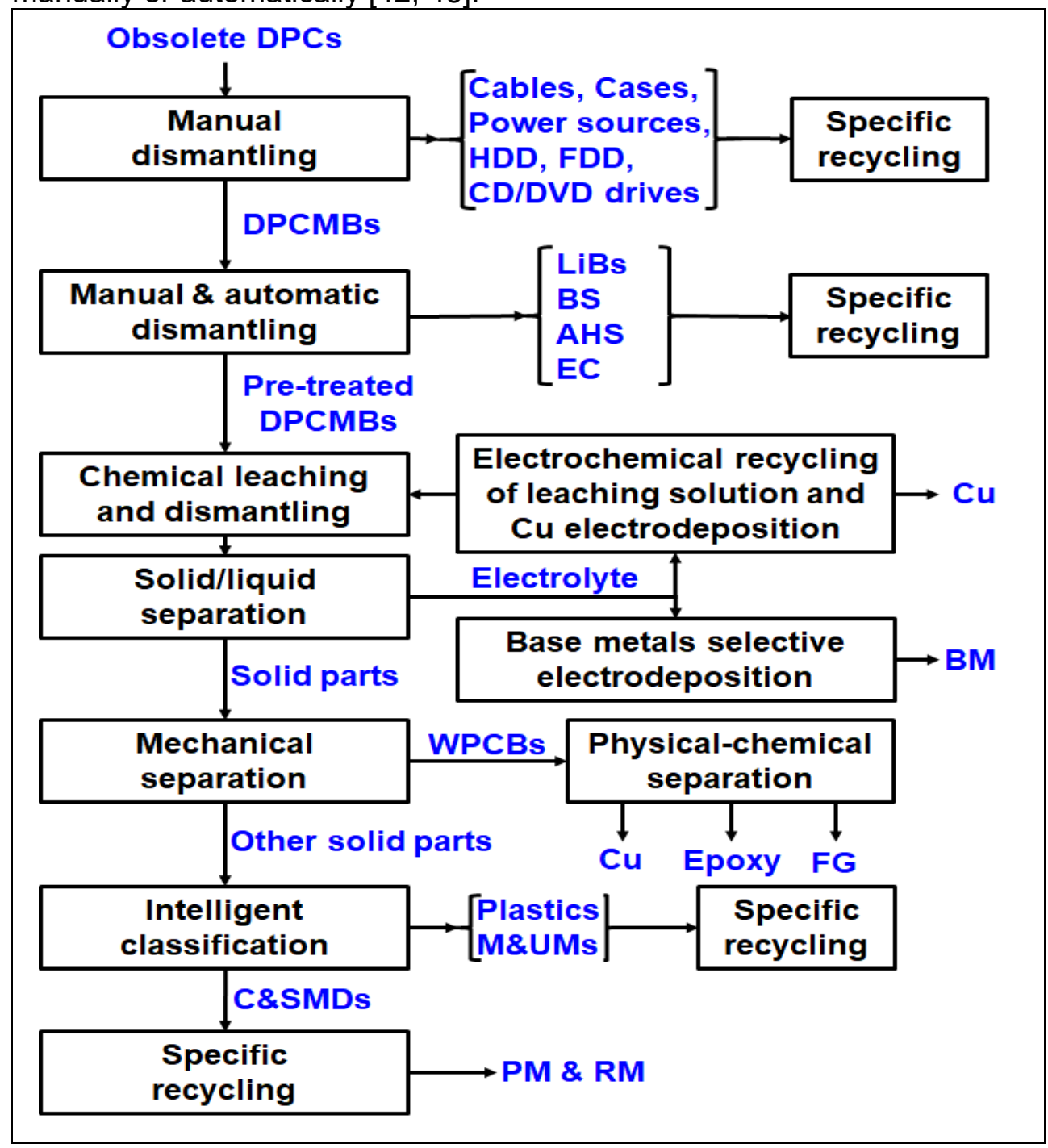

Figure 5. Proposed flowchart for the complete recycling of DPCMBs waste 
The exposed metals from the whole pre-treated DPCMBs can be dissolved in appropriated equipment using electrochemically regenerated leaching solutions [26], preserving, in this manner, unaltered the solid parts. A simple mechanical separation (sieving) allows the separation of the depopulated WPCBs that can be easier recycled to recover pure $\mathrm{Cu}$, epoxy and fiberglass (FG) [44]. Subsequently, an intelligent (robotized) separation can be applied to the remaining solid parts as electronic components (C\&SMDs), M\&UMs and plastics, the last ones able to be sorted depending on shape, composition or even color [45] and valorized superiorly with high revenues [46]. Finally, the C\&SMDs fraction, containing the major amount of PM and rare metals (RM) can be appropriate recycled for their efficient recovering [47].

\section{CONCLUSIONS}

Analyzing the results concerning the composition evolution of 10 different DPCMBs released between 1998 and 2008, we concluded that the amounts of metallic and non-metallic components present, generally, a random distribution, without any evident tendency of evolution or involution. Consequently, using the mean values of the included components amounts, we estimated that the appropriate recycling of the contained metals leads to a revenue of more than $7000 €$ for 1 ton of recycled DPCMBs. Also, the WPCBs and plastic fractions, representing more than $60 \%$ of the DPCMBs total mass, must be carefully treated to recover supplementary materials and avoid the generation of new wastes fluxes.

Finally, combining the literature information with the practical observations we were able to propose an original flowchart combining multiples technologies able to recover ALL the components included in the DPCMBs structure. Such technology is in total agreement with the new imposed circular economy concept, leading to complete materials recycling without, theoretically, any production of secondary wastes.

\section{EXPERIMENTAL SECTION}

The starting materials for this study were represented by the obsolete CPUs discarded from the Faculty of Chemistry and Chemical Engineering (Babeş-Bolyai University, Cluj-Napoca, Romania) in 2016. Around 30 CPUs were disassembled manually, and the resulting components were sorted on different categories: cables, power sources, floppy disk and optical units, HDDs, microprocessors heat sinks systems, DPCMBs, microprocessors, RAM modules and dedicated cards (video, sound, network, data acquisition, etc.). From the resulting stock, 10 DPCMBs released between 1998 and 2008 were manually pre-treated to 
remove the LiBs, AHSs, BSs and ECs. The weight of the DPCMBs before and after the pre-treatment and, also, of the dismounted components was evaluated using a laboratory electronic balance (model PLJ-510-3m, KERN\&SOHN GmbH, Germany). Subsequently, each board was cut in large pieces (around $5 \mathrm{~cm} \times 10 \mathrm{~cm}$ ), introduced in sealable glass pots of 3$5 \mathrm{~L}$ and the exposed metals were leached for $48 \mathrm{~h}$ using, for each sample, $2.2 \mathrm{~L}$ of $2 \mathrm{M} \mathrm{KBr}$ solution containing $100 \mathrm{~mL} \mathrm{HCl} 37 \%$ and $100 \mathrm{~mL}$ liquid $\mathrm{Br}_{2}$ as oxidizing agent. After the leaching step, the resulting solutions were decanted, and the remaining solid components were washed with tap and distilled water, dried for in air 7 days, sorted manually as WPCBs, PPs, C\&SMDs and M\&UMs, and weighed separately. The main information concerning the selected DPCMBs samples are indicated in Table 2.

Table 2. The assigned experimental code, model, release year and the initial weight of the selected DPCMBs samples

\begin{tabular}{|c|l|c|c|}
\hline Sample code & \multicolumn{1}{|c|}{ Model } & Release year & Initial weight $(\mathrm{g})$ \\
\hline S01-1998 & Asus P2B-S & 1998 & 683.1 \\
\hline S02-1999 & PRM-00801 E1 & 1999 & 554.6 \\
\hline S03-2000 & PRM-27IV- DTK Computer & 2000 & 520.5 \\
\hline S04-2002 & MSI MS-6378 & 2002 & 530.7 \\
\hline S05-2003 & Elitegroup K7VTA3/KT333 & 2003 & 657.1 \\
\hline S06-2003 & Elitegroup P4IBASD (v3.0) & 2003 & 646.4 \\
\hline S07-2004 & ASRock P4i45D+ & 2004 & 539.1 \\
\hline S08-2004 & Mercury KOB KT266a FDSX & 2004 & 660.3 \\
\hline S09-2006 & ASRock AliveNF6G-VSTA & 2006 & 535.8 \\
\hline S10-2008 & MSI P31 Neo V2 & 2008 & 573.8 \\
\hline
\end{tabular}

The resulting leaching solutions were filtered, diluted (depending on necessities, of 10, 100 or 1000 times) and analyzed by ICP-OES using a SPECTRO CIROS CCD spectrometer (SPECTRO Analytical Instruments, Germany).

In order to evaluate the metals content in the C\&SMDs fraction, a $15 \mathrm{~g}$ homogenous sample of chips and SMD parts was calcined at $800^{\circ} \mathrm{C}$ (Heraeus $\mathrm{GmbH}$, Germany) until constant weight, finely grinded and digested with $55 \mathrm{~mL}$ of aqua regia. From the resulting solution, the amount of $\mathrm{Cu}, \mathrm{Zn}, \mathrm{Ni}, \mathrm{Sn}, \mathrm{Fe}$, $\mathrm{Ag}, \mathrm{Au}$ and Pb was evaluated by F-AAS (AVANTA PM, GBC, Australia).

\section{ACKNOWLEDGMENTS}

This work was supported by a grant of the Romanian Ministry of Research and Innovation, CCCDI-UEFISCDI, project number PN-III-P1-1.2PCCDI-2017-0652 / 84PCCDI/2018, within PNCDI III. 


\section{REFERENCES}

1. https://www.statista.com/statistics/272595/global-shipments-forecast-for-tabletslaptops-and-desktop-pcs/, accessed 21.11.2018.

2. A. Puca, M. Carrano, G. Liu, D. Musella, M. Ripa, S. Viglia, S. Ulgiati, Resources, Conservation and Recycling, 2017,116, 124.

3. S.S. Suresh, S. Bonda, S. Mohanty S.K. Nayak, Process Safety and Environmental Protection, 2018, 116, 477.

4. G. Chauhan, P.R. Jadhao, K.K. Pant, K.D.P. Nigam, Journal of Environmental Chemical Engineering, 2018, 6(1), 1288.

5. C.A. Kohl, L. P. Gomes, Journal of Cleaner Production, 2018, 184, 1041.

6. Z. Wu, W. Yuan, J.Li, X. Wang, L. Liu, J. Wang, Frontiers of Environmental Science and Engineering, 2017, 11(5):8, 1.

7. M. Kaya, "Recovery of Metals and Nonmetals from Waste Printed Circuit Boards (PCBs) by Physical Recycling Techniques" in Energy Technology 2017, L. Zhang (Ed.), The Minerals, Metals \& Materials Society, 2017, 433-451.

8. S. Pinho, M. Ferreira, M. F. Almeida, Resources, Conservation and Recycling, 2018, 132, 71.

9. A. Barnwal, S. Vishvakarma, N. Dhawan, Materials Today: Proceedings, 2018, 5(9), 17046.

10. H. Li, J. Eksteen, E. Oraby, Resources, Conservation and Recycling, 2018, 139, 122.

11.B. Mizero, T. Musongo, E.R. Rene, F. Battes P.N.L. Lens, Process Safety and Environmental Protection, 2018, 120, 14.

12.H. Wang, S. Zhang, B. Li, D. Pan, Y. Wu, T. Zuo, Resources, Conservation and Recycling, 2017, 126, 209.

13.M. Ghodrat, M.A. Rhamdhani, A. Khaliq, G. Brooks, B. Samali, Journal of Material Cycles and Waste Management, 2018, 20(1), 386.

14.A. Gurgul, W. Szczepaniak M. Zabłocka-Malicka, Science of the Total Environment, 2018, 624, 1119.

15. A. Priya, S. Hait, Environmental Science and Pollution Research, 2017, 24(8), 6989.

16.E. Ventura, A. Futuro, S.C. Pinho, M.F. Almeida, J.M. Dias, Journal of Environmental Management, 2018, 223, 297.

17.M. Kaya, Waste Management, 2016, 57, 64.

18.A. Işıldar, E.R. Rene, E.D. van Hullebusch, P.N.L. Lens, Resources, Conservation and Recycling, 2018, 135, 296.

19.C. Ning, C.S.K. Lin, D.C. W. Hui, G. McKay, Topics in Current Chemistry, 2017, 375(2), 1.

20.G. Cecere, A. Martinelli, Research Policy, 2017, vol. 46(5), 925.

21. L. Rocchetti, A. Amato, F. Beolchini, Journal of Cleaner Production, 2018,178, 814.

22. G. Zhang, Y. He, Y. Feng, T. Zhang, H. Wang, X. Zhu, Separation and Purification Technology, 2018, 207, 321.

23.R. Saini, R. Khanna, R.K. Dutta, R. Cayumil, M. Ikram-UI-Haq, V. Agarwala, G. Ellamparuthy, K. Jayasankar, P.S. Mukherjee, V. Sahajwalla, Waste Management, 2017, 64, 182. 
24.S. Fogarasi, F. Imre-Lucaci, Á. Imre-Lucaci, and P. Ilea, Journal of Hazardous Materials, 2014, 273, 215.

25.L.A. Diaz, T.E. Lister, J.A. Parkman, G.G. Clark, Journal of Cleaner Production, 2016, 125, 236.

26.S.A. Dorneanu, Studia Universitatis Babes-Bolyai Chemia, 2017, 62(3), 177.

27.D. Yang D. Yang, Y. Chu, J. Wang, M. Chen, J. Shu, F. Xiu, Z. Xu, S. Sun, S. Chen, Separation and Purification Technology, 2018, 205, 302.

28.E. Haccuria, P. Ning, H. Cao, P. Venkatesan, W. Jin, Y. Yang, Z. Sun, Journal of Cleaner Production, 2017, 152, 150.

29.L. A. Diaz, T. E. Lister, Waste Management, 2018, 74, 384.

30.S. Fogarasi, F. Imre-Lucaci, A. Egedy, Á. Imre-Lucaci, P. Ilea, Waste Management, 2015, 40, 136.

31.K. Scott, Renewable and Sustainable Energy Reviews, 2018, 81, 1406.

32. Y. Zhang, M. Chen, Q. Tan, B. Wang, S. Chen, Hydrometallurgy, 2018, 175, 150.

33. S.M. Abdelbasir, C.T. El-Sheltawy, D.M. Abdo, Journal of Sustainable Metallurgy, 2018, 4(2), 295.

34.J.R. Peeters, P. Vanegas, W. Dewulf, J.R. Duflou, Journal of Cleaner Production, 2017, 140, 1182.

35. A. Cesaro, A. Marra, V. Belgiorno M. Guida, Journal of Cleaner Production, 2017, $142,2656$.

36.R.G. Charles, P. Douglas, I.L. Hallin, I. Matthews, G. Liversage, Waste Management, 2017, 60, 505.

37.https://ec.europa.eu/eurostat/statisticsexplained/index.php/Electricity_price_statistics, accessed 21.11.2018.

38.http://www.nanotech-elektronik.pl/index.php/en/42-english/support, accessed 21.11.2018.

39.R.A. Mesquita, R.A.F. Silva, D. Majuste, Process Safety and Environmental Protection, 2018, 120, 107.

40.https://www.Ime.com/Metals, accessed 21.11.2018.

41.F. Tesfaye, D. Lindberg, J. Hamuyuni, P. Taskinen, L. Hupaa. Minerals Engineering, 2017, 111, 209.

42.B. Kopacek, IFAC-PapersOnLine, 2016, 49(29), 190.

43.J. Li, M. Barwood, S. Rahimifard, Resources Conservation and Recycling, 2019, 140, 158.

44.H.R. Verma, K.K. Singh, T.R. Mankhand, Waste Management, 2017, 65, 139.

45.S.P. Gundupalli, S. Hait, A. Thakur, Waste Management, 2017, 60, 56.

46.R. Gao, Z. Xu, Journal of Hazardous Materials, 2019, 364, 1.

47.Y. Liu, K, Li, J. Guo, Z. Xu, Journal of Cleaner Production, 2018, 197, 1488. 